\title{
A fast intensity simulator for tropical cyclone risk analysis
}

\author{
Kerry Emanuel ${ }^{1}$
}

Received: 4 February 2017/Accepted: 21 April 2017/Published online: 6 May 2017

(C) The Author(s) 2017. This article is an open access publication

\begin{abstract}
Robust estimates of tropical cyclone risk can be made using large sets of storm events synthesized from historical data or from physics-based algorithms. While storm tracks can be synthesized very rapidly from statistical algorithms or simple dynamical models (such as the beta-and-advection model), estimation of storm intensity by using full-physics models is generally too expensive to be practical. Although purely statistical intensity algorithms are fast, they may not be general enough to encompass the effects of natural or anthropogenic climate change. Here we present a fast, physically motivated intensity algorithm consisting of two coupled ordinary differential equations predicting the evolution of a wind speed and an inner core moisture variable. The algorithm includes the effects of ocean coupling and environmental wind shear but does not explicitly simulate spatial structure, which must be handled parametrically. We evaluate this algorithm by using it to simulate several historical events and by comparing a risk analysis based on it to an existing method for assessing long-term tropical cyclone risk. For simulations based on the recent climate, the two techniques perform comparably well, though the new technique does better with interannual variability in the Atlantic. Compared to the existing method, the new method produces a smaller increase in global tropical cyclone frequency in response to global warming, but a comparable increase in power dissipation.
\end{abstract}

Keywords Tropical cyclones · Risk modeling · Hurricanes ·

Hurricane intensity

\section{Introduction}

Tropical cyclones are a leading cause of mortality and property loss worldwide (EM-DAT 2016); thus, quantitatively accurate assessment of tropical cyclone risk is of great interest to governments and industries in affected regions. But historical cyclone records are

Kerry Emanuel

emanuel@mit.edu

1 Lorenz Center, Massachusetts Institute of Technology, Room 54-1814, MIT, 77 Massachusetts Avenue, Cambridge, MA 02139, USA 
generally too short and inaccurate for direct use in risk assessment and so contemporary methods of risk assessment rely either on bootstrapping historical storm records or on physics-based approaches. For example, the leading industry risk models are based on large sets of synthetic storm tracks generated from the statistics of historical tracks (Vickery et al. 2000; Yonekura and Hall 2011) and with intensity evolutions that are based on observed intensities and their relationships to environmental predictors such as sea surface temperature. Such models are heavily empirical and thus largely constrained to tropical cyclone climatology over the period of the historical tropical cyclone record. Thus, it is difficult to account for the effects of climate change, whether natural or anthropogenic, on time scales too long to have been well sampled in the historical tropical cyclone record.

To circumvent these limitations of historically based risk assessment, efforts have been made in recent years to simulate tropical cyclones using physics-based models driven by the large-scale climate conditions provided by global reanalysis data and/or by global climate models. For example, Emanuel et al. (2006) generated synthetic hurricane tracks using a beta-and-advection model (Marks 1992) driven by large-scale environmental flow synthesized from global climate reanalysis data, comparing the results to a Markov-chain approach based on historical storm tracks. Genesis points in both cases were drawn from probability density analyses based on historical genesis locations, and intensities were calculated using a simple coupled ocean-atmosphere tropical cyclone model (CHIPS; Emanuel et al. 2004). Likewise, Colbert et al. (2013) used a beta-and-advection model to synthesize tropical cyclone tracks from large-scale flow statistics, though they did not try to predict intensity. Emanuel et al. (2008) developed a synthetic event generator entirely free from historical storm data. They generated storms by randomly seeding large-scale climate states and then using the CHIPS intensity model to determine which seeds survive, tracking the events using the beta-and-advection model driven by the large-scale environmental flow, as before. Because such a method is based purely on physics applied to large-scale environmental conditions, it can be driven by climate model output as well as by global reanalysis data, allowing for quantification of the effects of climate variability and trends on tropical cyclone genesis, tracks, and intensity.

Although the CHIPS model is far faster than full-physics tropical cyclone models of the kind used in operational tropical cyclone forecasting, it is still the slowest component of the aforementioned physics-based tropical cyclone event generator. Yet its generality and applicability to different climate states make it an attractive alternative to purely statistical approaches. Might it be possible to develop a physically based algorithm that is nearly as accurate and general as CHIPS but with speeds approaching that of purely statistical methods? We here present such an algorithm. We begin with a description of the method and proceed to test its ability to simulate individual historical events and to replicate key historical tropical cyclone statistics.

\section{Tropical cyclone intensity simulator}

The intensity simulator is motivated by experience with CHIPS and deductions from a nonlinear analytical model. An earlier version that does not explicitly include the effects of the pressure dependence of the surface saturation specific humidity or dissipative heating ${ }^{1}$ is described in Emanuel and Zhang (2017).

\footnotetext{
1 As air flows toward the storm center, the air pressure drops and this increases the amount of water that can be evaporated into the air. Also, the friction that accompanies the strong turbulence in the inflow heats it; this is known as "dissipative heating".
} 
Emanuel (2012) developed a nonlinear analytical model of the intensification of a tropical cyclone whose inner core is fully water saturated and for which the effects of environmental wind shear, the pressure dependence of the surface saturation specific humidity, and dissipative heating in inflowing boundary layer air are ignored. An approximation to the full equations of that model leads to an intensity equation that takes the form

$$
\frac{\mathrm{d} V}{\mathrm{~d} t} \cong \frac{C_{\mathrm{D}}}{2 h}\left(V_{p 0}^{2}-V^{2}\right)
$$

where $V_{p 0}$ is the potential intensity ${ }^{2}$ defined without accounting for dissipative heating or the pressure dependence of the saturation mixing ratio, $V$ is the maximum circular (tangential) wind speed, $C_{\mathrm{D}}$ is the surface drag coefficient, $h$ is a boundary layer depth, and $V_{p}$ is a base potential intensity (not including the pressure dependence of the saturation mixing ratio or dissipative heating) modified by a function of the surface exchange coefficients of enthalpy and momentum.

We first seek a set of equations that reduce to (1) in the limit of a fully water saturated core $^{3}$ free of wind shear but which incorporates the effects of an unsaturated inner core, environmental wind shear, and ocean interaction and which behaves qualitatively like the full CHIPS model. After much experimentation, we developed a pair of ordinary differential equations for the surface circular wind speed $V$ and a nondimensional inner core moisture variable $m$ that varies from 0 to 1 and can be thought of as a kind of relative humidity:

$$
\frac{\mathrm{d} V}{\mathrm{~d} t}=\frac{1}{2} \frac{C_{\mathrm{D}}}{h}\left[\alpha \beta V_{p}^{2} m^{3}-\left(1-\gamma m^{3}\right) V^{2}\right]
$$

and

$$
\frac{\mathrm{d} m}{\mathrm{~d} t}=\frac{1}{2} \frac{C_{\mathrm{D}}}{h}[(1-m) V-2.2 S m],
$$

where $V_{p}$ is the full potential intensity, $S$ is the magnitude of the $850-250 \mathrm{hPa}$ environmental wind shear, $\alpha$ is an ocean interaction parameter that varies from zero to one, and $\beta$ and $\gamma$ are dimensionless parameters to be discussed presently. [In (2) and (3), the units of $V$, $V_{p}, S$, and $h$ must be consistent.]

The ocean feedback parameter is modeled after the results of Schade and Emanuel (1999) and is intended to account for the strong cooling of the sea surface induced by the stirring up of cold, deep waters by the tropical cyclone's surface winds. They coupled the CHIPS model to a three-dimensional ocean model and performed a large set of experiments with different upper ocean thermal structures, storm dimensions, and storm translations speeds and found a good curve fit that accurately predicts the final, steady intensity of the simulated storms. We use an approximation to that curve fit here to define the parameter $\alpha$ that appears in (2):

$$
\alpha=1-0.87 e^{-z}
$$

where

\footnotetext{
2 The potential intensity is the maximum storm-relative surface wind speed that is theoretically possible in a tropical cyclone, given the ocean and atmospheric temperatures.

3 The humidity of the innermost $150 \mathrm{~km}$ of tropical cyclones, usually referred to as their "cores," is known to have an important effect on their rates of intensification.
} 


$$
z \equiv 0.01 \Gamma^{-0.4} h_{m} u_{T} V_{p} V^{-1}
$$

Here $\Gamma$ is the sub-mixed layer thermal stratification in $K(100 m)^{-1}, h_{m}$ is the ocean mixed layer depth in meters, and $u_{T}$ is the storm translation speed in $m s^{-1}$. Inspection of (4) and (5) shows that the effective potential will be larger ( $\alpha$ closer to unity) when storms are weaker or move faster, the mixed layer is deeper, or the thermal stratification below the mixed layer is weaker.

The Schade and Emanuel (1999) formulation was developed to describe the steady-state intensity of idealized storms translating at constant speed in a constant atmospheric and oceanic thermal environment. While it seems reasonable to suppose that this is a good "target" intensity to use in (2), that is no guarantee that the effects of ocean feedback on intensity change will be handled correctly.

Emanuel and Zhang (2017) show that the term 2.2Sm that appears in (3) is the equivalent of the ventilation $v$ introduced by Tang and Emanuel (2010) and that the system consisting of the steady-state forms of (2) and (3) with no ocean interaction $(\alpha=1)$ has the same qualitative stability behavior as the steady-state model they developed.

There are at two possible ways to incorporate the effects of dissipative heating and the pressure dependence of the saturation mixing ratio. The simplest way, employed by Emanuel and Zhang (2017), is just to use in (2) the full form of the potential intensity that incorporates isothermal expansion and dissipative heating. For storms that are near their potential intensity, this should be adequate. But for much weaker storms, the isothermal expansion and dissipative heating are correspondingly weaker and this is not accounted for by using the full potential intensity in (2).

In "Appendix," we show that dissipative heating and the pressure dependence of the surface saturation mixing ratio can be accounted for by continuing to use the full potential intensity in (2), as in Emanuel and Zhang (2017), and taking

$$
\beta=1-\epsilon-\kappa,
$$

and

$$
\gamma=\epsilon+\alpha \kappa
$$

where

$$
\epsilon \equiv \frac{T_{\mathrm{s}}-T_{\mathrm{o}}}{T_{\mathrm{s}}}
$$

is the thermodynamic efficiency, and

$$
\kappa \equiv \frac{\epsilon}{2} \frac{C_{k}}{C_{D}} \frac{L_{\mathrm{v}} q_{0}^{*}}{R_{d} T_{\mathrm{s}}} .
$$

Here $T_{\mathrm{s}}$ is the surface temperature, $T_{\mathrm{o}}$ is the tropical cyclone outflow temperature, $C_{k}$ is the surface enthalpy exchange coefficient, $L_{\mathrm{v}}$ is the latent heat of vaporization, $q_{0}^{*}$ is the surface saturation specific humidity at ambient environmental surface pressure, and $R_{d}$ is the gas constant for dry air.

Note that (2) and (3) reduce to the form of (1) when $m=1$ and there is no ocean interaction $(\alpha=1)$ or environmental wind shear.

Thus, our system is comprised of (2) and (3) with the definitions (4)-(9). While the development of the equations was guided by existing theory and models, they should be regarded mostly as an empirical construct, lying somewhere between formal, deterministic 
models and purely statistical models. They were designed and tested to mimic the behavior of CHIPS, both as it is used in real-time forecasting and as a component of a tropical cyclone risk model.

To run this system, it is necessary to specify the potential intensity, magnitude of the 250-850 hPa environmental wind shear, ocean mixed layer depth, and ocean sub-mixed layer thermal stratification along the track of the storm as well as the storm's translation speed. The sea surface temperature and outflow temperature along the track are also needed for the specification of $\beta$ and $\gamma$ according to (6)-(9), but we approximate these by constants in the test results to be discussed presently. Initial conditions for the circular surface wind speed $V$ and moisture variable $m$ also need to be specified.

We next present comparisons between CHIPS and the intensity simulator.

\section{Comparison to CHIPS hindcasts}

The CHIPS model has been used to make automated intensity forecasts every year for about the past 15 years. The system completes nearly every 6-h forecast cycle beginning when the observed event first attains a surface wind speed of $35 \mathrm{kts}$. (A few cycles are missed owing to missing data or inoperative computers.) The system uses the National Centers for Environmental Prediction (NCEP) Global Forecasting System (GFS) operational analyses and forecasts to calculate the environmental shear and potential intensity of the initial state and along the forecast track. (Shear associated with the GFS-modeled tropical cyclone is first removed from the analyses and forecasts before calculating the environmental shear.) We record the modeled intensity and all the environmental inputs every $2 \mathrm{~h}$ of the forecast.

After the end of the event, we can perform hindcasts that use the observed (rather than forecast) storm positions and the 0-h environmental variables from each 6-h initialization time. These hindcasts thus used the best reconstruction of the actual storm track and the operationally analyzed wind shear and potential intensity bilinearly interpolated to the storm position.

Ocean mixed layer depth and sub-mixed layer stratification are derived from monthly mean climatology (Levitus 1982) and linearly interpolated to the storm date and time and bilinearly to its position. (One could potentially improve on this by using operational analyses or reanalyses of actual upper ocean conditions.) One-quarter degree bathymetry and topography are used to determine when a storm makes landfall, at which time the surface turbulent enthalpy flux is switched off and the surface drag coefficient is increased. Also, when and where the ocean mixed layer depth exceeds the local ocean depth, it is assumed that the ocean is well mixed to the bottom and sea surface cooling by mixing is shut off.

For the CHIPS hindcasts presented here, we initialize the model's wind and humidity fields by setting the initial circular wind speed equal to the observed storm intensity and by continuously adjusting the model's middle tropospheric humidity variable during the first $24 \mathrm{~h}$ of integration so as to drive the model's wind intensity toward the observed intensity. Thereafter, the model is run freely. Since the initial rate of intensification is sensitive to inner core middle tropospheric water vapor, this initialization procedure has the effect of initializing both the intensity and the inner core moisture. It takes advantage of the fact that wind intensity is generally much better observed than inner core moisture. The importance of inner core moisture initialization is discussed in Emanuel and Zhang (2017). 
In initializing the wind speed, we first subtract a fraction of the storm translation speed to estimate the storm-relative circular wind component we are actually integrating.

We apply the same environmental shear, potential intensity, ocean mixed layer properties, and bathymetry to the integration of the tropical cyclone intensity simulator presented here. The potential intensity in (2) is set to zero upon landfall as in the CHIPS model, and likewise ocean mixing is turned off when the mixed layer depth equals or exceeds the local ocean depth.

For the present purpose, we set $\epsilon$ and $\kappa$ to constants but retain variable $\alpha$ in (2) and (7). Specifically, we set $C_{\mathrm{D}}=1.2 \times 10^{-3}, h=1400 \mathrm{~m}, \epsilon=0.33$, and $\kappa=0.1$. Here again, once might be able to improve the model by incorporating a more realistic formulation of the surface drag as well as variable values of $\epsilon$ and $\kappa$.

As with CHIPS, we initialize $V$ with the best track wind speed early in the storm's life, minus a fraction of the storm translation speed as a way of estimating the storm-relative circular wind component. But we take a different approach to initializing the moisture variable $m$ : We simply invert (2) to find $m$ given the change in the best track intensity over the 6-h after initialization. This insures that the inner core moisture variable is initialized so as to yield the observed initial rate of intensification. In effect, we are taking as initial conditions the observed circular wind $V$ and its rate of change, $\mathrm{d} V / \mathrm{d} t$. But note that the CHIPS hindcasts have an advantage over the present tropical cyclone intensity simulator: Its initialization procedure drives the modeled storm intensity toward the observed intensity over the first $24 \mathrm{~h}$.

We employ a conventional leap-frog time integration scheme with an Asselin filter value of 0.1 and a time step of $240 \mathrm{~s}$. An integration over a typical storm track takes about $0.002 \mathrm{~s}$ on a conventional laptop. Thus, it is feasible to simulate the intensities of very large numbers of tropical cyclones, given their tracks and large-scale environments.

Figure 1 shows a representative example: Atlantic Hurricane Ivan of 2004. The overall behavior of the hindcasts of the two models is rather similar, and the mean absolute errors of the two are nearly the same. Figure 2 shows the mean absolute errors accumulated over the lifetimes of each Atlantic hurricane of the 2004 season. Accumulated over the whole season, the mean absolute error of the CHIPS hindcasts is $19.0 \mathrm{kts}$ while that of the intensity simulator is $17.8 \mathrm{kts}$; thus, judging from this single season, the two methods are competitive. Our purpose here is merely to show that the simulator produces reasonable results when run in hindcast mode; evaluating the actual forecast skill of the simulator is deemed beyond the scope of the current study. We next turn to an evaluation of how well the simulator performs as part of a tropical cyclone risk model.

\section{Performance in a risk model}

The main motivation for developing a fast intensity simulator is to provide a faster, simpler way of simulating large numbers of events to assess tropical cyclone event risk. Here we substitute the intensity simulator for the CHIPS model in the event risk model described in detail in Emanuel et al. (2006, 2008). A summary of the technique is also provided in Emanuel and Zhang (2016). Broadly, monthly mean winds and their variances and covariances, all generated from reanalyses or global climate models, are used to generate synthetic time series of winds that have the correct monthly means, variances, and covariances and have power spectra that fall off as frequency cubed, similar to observed flows at synoptic and planetary scales. Tropical cyclone tracks are generated by seeding 


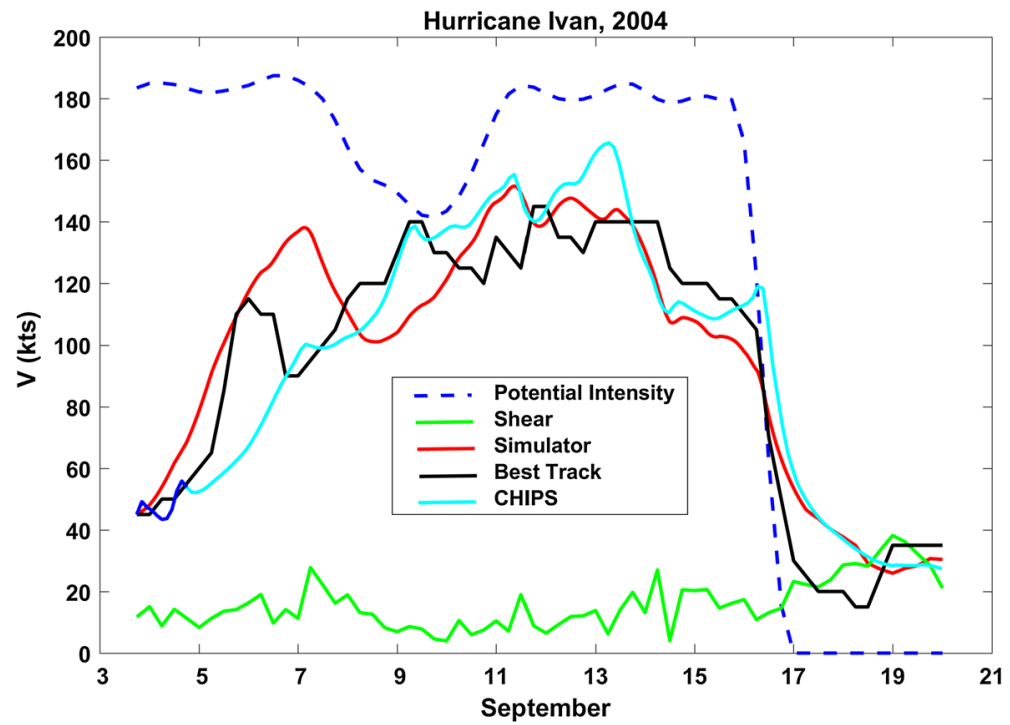

Fig. 1 Hindcast simulations of the intensity of Atlantic Hurricane Ivan of 2004 by the CHIPS model (cyan) and the intensity simulator ( $r e d$ ), compared to the best track observed intensity (black). The dark blue segment of the CHIPS simulation denotes the initialization period. Also shown are the along-track potential intensity (dashed blue) and environmental 850-250 hPa shear (green)

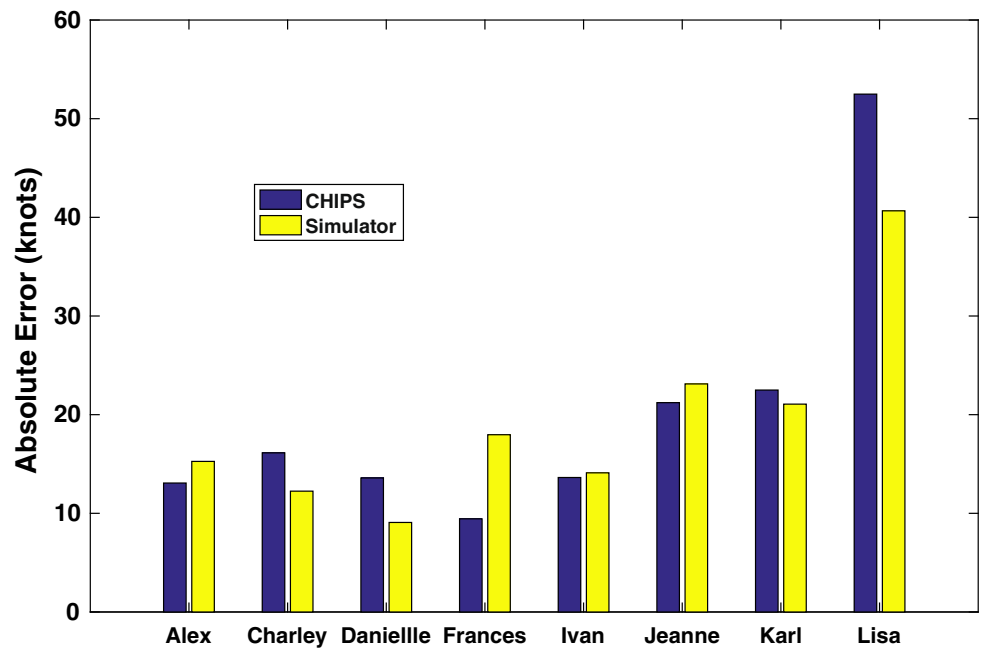

Fig. 2 Comparison of mean absolute error (kts) accumulated over the lifetimes of each Atlantic hurricane of the 2004 season, showing CHIPS (blue) and the present intensity simulator (yellow)

randomly in space and time with weak protovortices which move with the synthesized winds with an additional component owing to the earth's sphericity and rotation. Then, the intensity model is run along each track, using the monthly mean thermodynamic environment (linearly interpolated in space and time) and climatological monthly mean upper ocean thermal conditions from reanalyses or climate models. The same winds used to 
generate the tracks provide the environmental shear that is an important component of the intensity model. The large majority of storms thus generated fail to intensify and are discarded. Only those cyclones that reach an intensity of at least $40 \mathrm{kt}$ are retained. The technique can therefore be regarded as working on the principle of natural selection.

The intensity simulator is driven using the same environmental variables that are used to drive CHIPS in the risk model: potential intensity, the magnitude of the environmental 850-250 hPa wind shear, ocean mixed layer depth, and sub-mixed layer thermal stratification. (In CHIPS, the ocean feedback is calculated interactively using a one-dimensional ocean model in which mixing is the only physical process simulated.)

The initial circular wind speed is likewise specified exactly as in the original risk model. However, the nondimensional moisture variable $m$ in the intensity simulator differs from the variable $\chi_{m}$ used in CHIPS. Here we set the initial value of $m$ to the $600 \mathrm{hPa}$ environmental relative humidity multiplied by 1.2 (but capped at unity) so that the initial seed disturbance has an inner core moisture that is elevated over that of the large-scale environment. There is no simple relationship between $m$ and the CHIPS variable $\chi_{m}$.

As with the original version of the risk model, we add a fraction of the translation velocity to the circular wind to determine the peak surface wind in each event at each time, following the methodology of Emanuel and Jagger (2010).

None of the comparisons we present here requires knowledge of the cyclone's structure or central pressure, but general applications do often require one or both. CHIPS is an axisymmetric model that does predict the evolution of the radial profile of wind and other quantities, but in practice we record only the radius of maximum surface wind and later fit canonical radial wind profiles to that radius and the maximum wind speed itself. To use the present intensity simulator, on the other hand, we must parameterize both the radius of maximum winds and the central pressure. We leave that to future work and focus here on comparisons that rely only on the maximum wind speed.

We produce 100 North Atlantic tropical cyclones in each year from 1979 to 2015 inclusive (3700 events in total) downscaled from NCAR/NCEP reanalyses (Kalnay et al. 1996), both for the original risk model and the version using the intensity simulator developed here. We shall hereafter refer to these as "CHIPS" and "FAST," respectively. The seeding rate is calibrated in both cases to yield the observed total number of tropical cyclones with lifetime maximum surface winds in excess of $40 \mathrm{kts}$, over the 37 -year period.

\subsection{Genesis}

A comparison of historical, CHIPS, and FAST genesis densities, calculated on a $4 \times 4$ degree latitude grid, is shown in Fig. 3. On the whole, the CHIPS model is closer to the observed distribution of genesis events. Both models have too few genesis events in the main development region of the tropical Atlantic east of the Caribbean, and both underpredict the rate of genesis to the east of Florida. This may be because the random seeding technique does not account for disturbances, such as African easterly waves, that often serve to initiate tropical cyclones in nature (Emanuel et al. 2008). CHIPS does a better job simulating the genesis minimum in the central Caribbean to the north of Venezuela. Although it here appears that CHIPS does a better job simulating the distribution of genesis, the difference between CHIPS and FAST is not as great as the difference between genesis fields downscaled from NCAR/NCEP reanalyses and fields downscaled from other reanalyses (e.g., ERA Interim; not shown). 

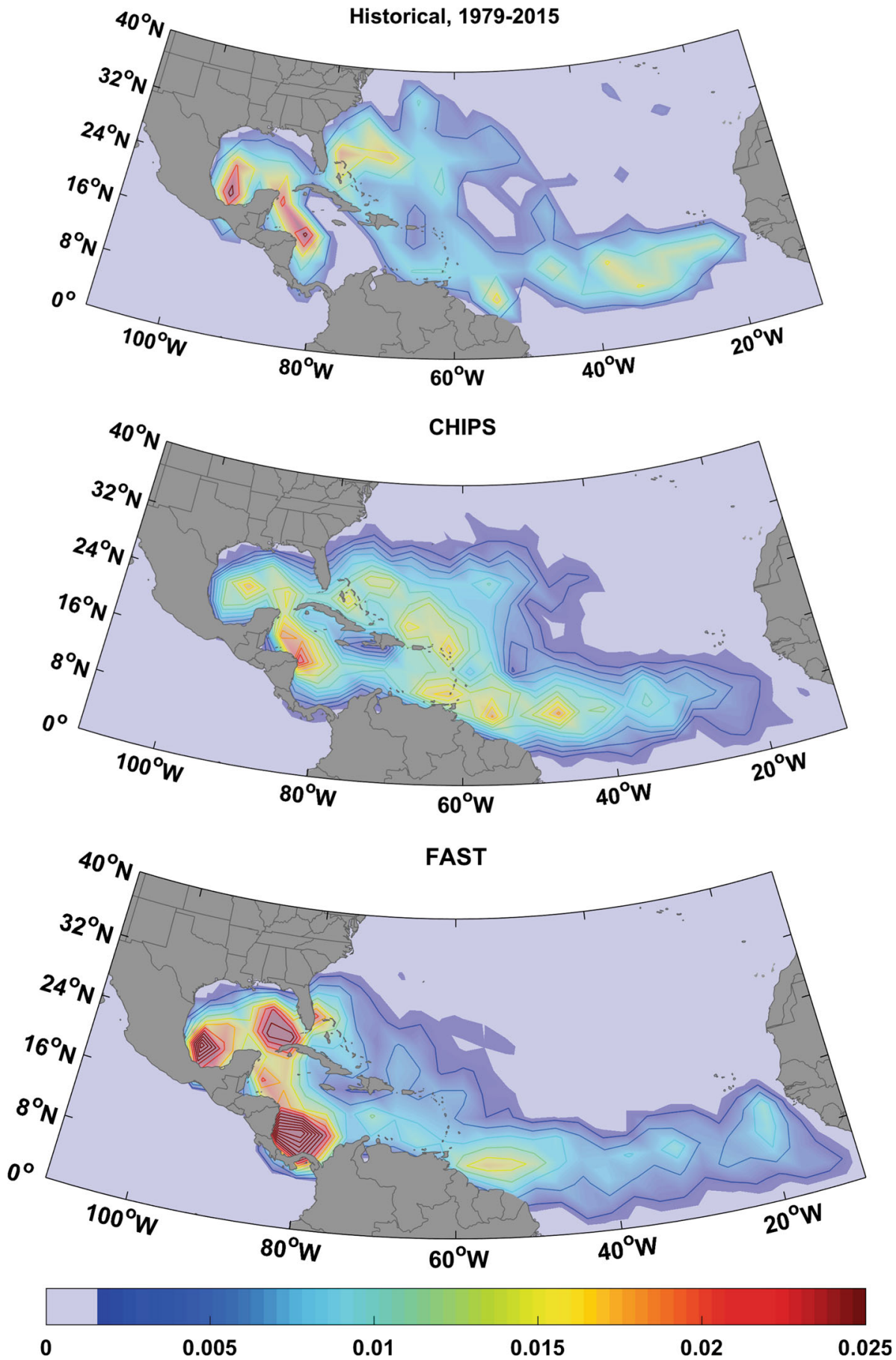

Fig. 3 Genesis densities, in number of genesis events per $1^{\circ}$ latitude square per year, from historical data (top), the original CHIPS-based risk model (center) and the new intensity simulator (bottom) 
Fig. 4 Number of tracks per 1 degree latitude square per year, from historical data (top), downscaled using CHIPS (center), and using FAST (bottom)

\subsection{Track density}

The number of track crossings per 2-degree latitude box is shown for historical data, CHIPS, and FAST in Fig. 4. The best track density is noisier because it is based on about 430 tracks, versus 3700 synthetic tracks. It is difficult to assess the relative quality of CHIPS and FAST, but they show clear differences.

\subsection{Intensity distribution}

The annual exceedance frequencies of storm lifetime maximum intensities from best track data are compared to those derived from the CHIPS and FAST versions of the risk model in Fig. 5. In general, there are slightly fewer FAST events than CHIPS events in a middle range (50-80 kts) and at the very highest range ( $>130 \mathrm{kts}$ ) of lifetime maximum intensity, but both fall within the $90 \%$ confidence intervals of being indistinguishable from the intensity distribution based on historical data. All three distributions are highly significantly different from each other according to two-sample Kolmogorov-Smirnov tests.

\subsection{Seasonal cycle}

The number of events in each month are shown for CHIPS and FAST and compared to historical data in Fig. 6. In general, CHIPS performs a little better, but the only truly significant difference is in the month of October, in which FAST overpredicts the frequency of events.

\subsection{Interannual variability}

Year-to-year variations in North Atlantic tropical cyclone frequency and storm lifetime maximum power dissipation are shown in Fig. 7. (The storm maximum power dissipation is the sum over all storms of the cube of their lifetime maximum wind speeds.) Here the FAST model outperforms CHIPS, especially in storm lifetime maximum power dissipation, where it accounts for about $68 \%$ of the best track variability compared to $37 \%$ with CHIPS. Figure 7 does show strong co-variability between the CHIPS and FAST model results.

\subsection{Response to global warming}

It is also of interest to compare the response of the new risk model to global warming. Emanuel et al. (2008) and Emanuel (2013) examined the response of the CHIPS-based risk model to warming produced under scenario A1B of the CMIP3 simulations and emission concentration pathway 8.5 in the CMIP5 models, respectively. The second study used 6 CMIP5 models, generating 600 events globally each year from 1950 to 2100 . Here we add a 7th model, the L'Institut Pierre-Simon Laplace IPSL-CM5A-LR model, to the original 6 and repeat the analyses with the FAST version of the risk model, driven by all 7 CMIP5 model outputs. (Owing to an oversight, we ran 500 rather than 600 events per year for the FAST simulations.) The 7 CMIP5 models are summarized in Table 1. 

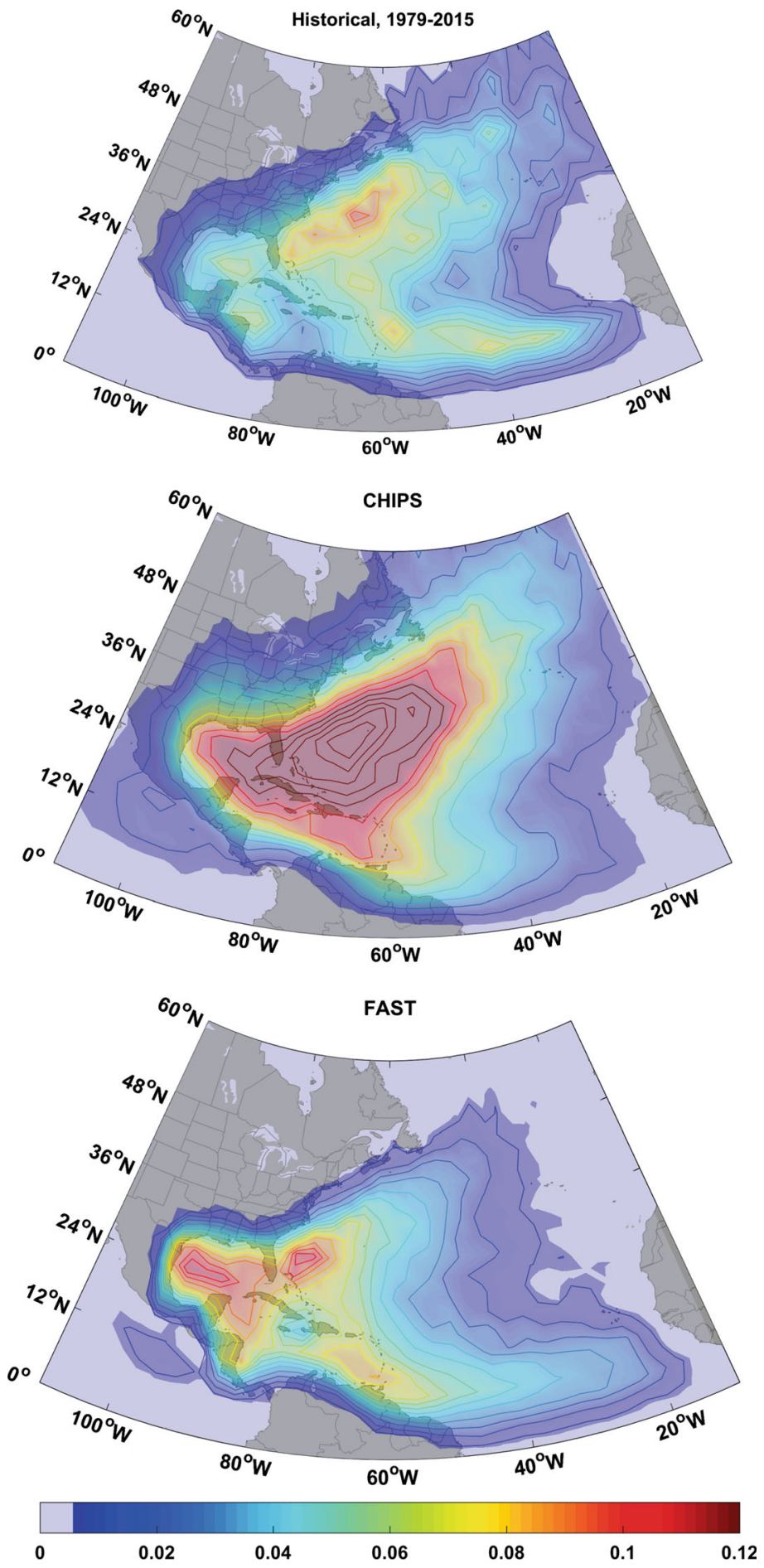


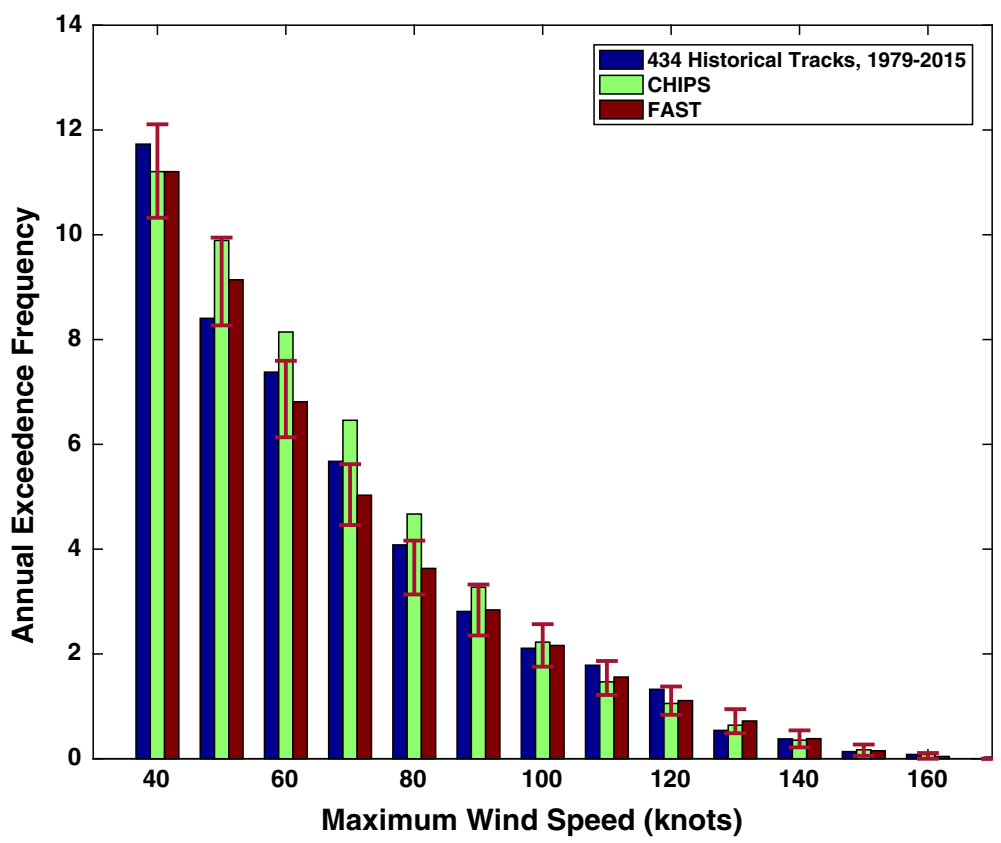

Fig. 5 Annual exceedance frequencies of storm lifetime maximum wind speeds for 434 historical tracks from 1979 to 2015 (blue), and 3700 CHIPS (green) and FAST (red) events. The error bars show limits within which $90 \%$ of subsamples of the FAST events of the size (434) of the best track events lie

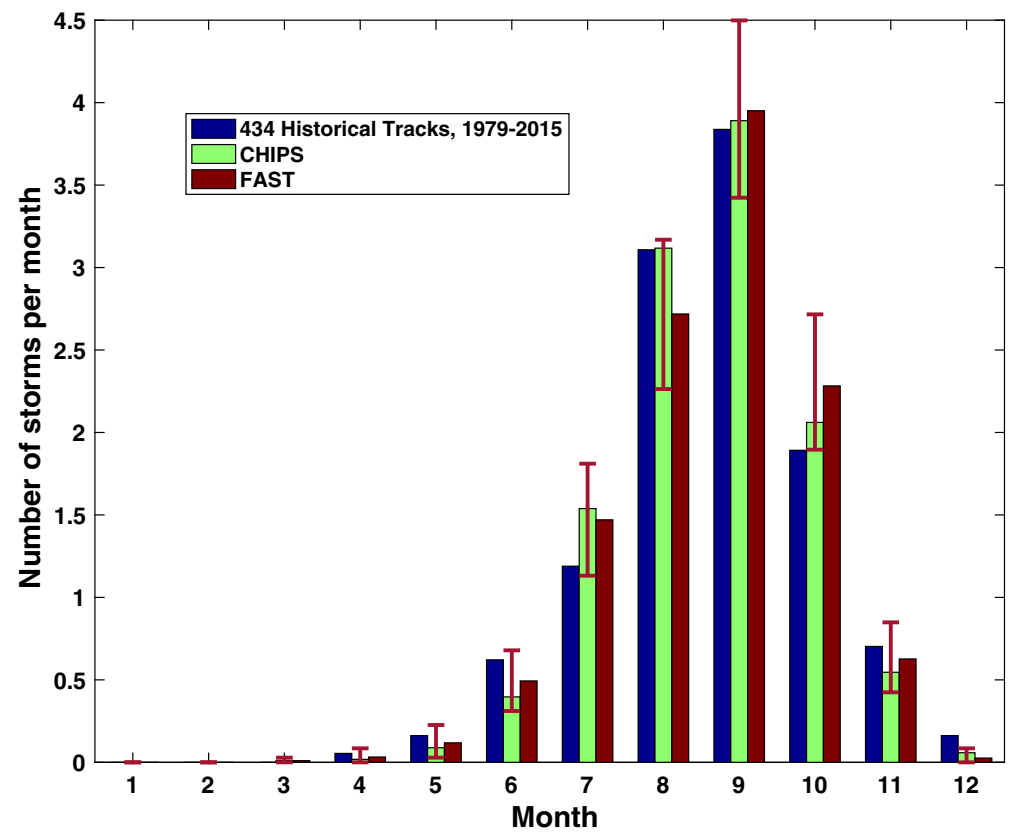

Fig. 6 Seasonal cycle of North Atlantic tropical cyclones from best track data (blue), and the CHIPS (green) and FAST (red) versions of the risk model. The dark red error bars show limits within which $90 \%$ of subsamples of the size of the best track data, drawn randomly from the FAST event set, lie 

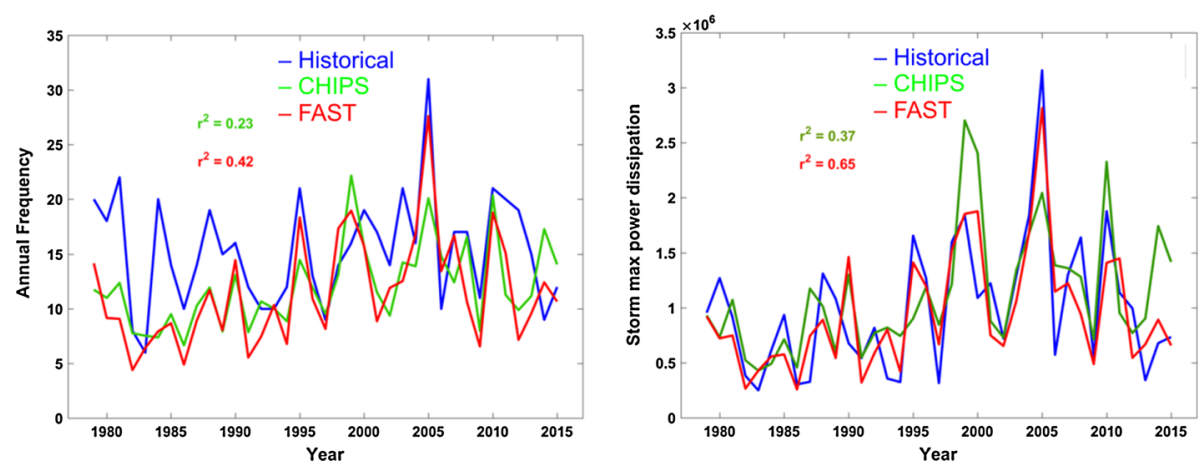

Fig. 7 Interannual variations of North Atlantic tropical cyclone frequency (left) and storm lifetime maximum power dissipation (right, in units of $\mathrm{m}^{3} \mathrm{~s}^{-3}$ ) from best track data (blue) and the CHIPS (green) and FAST (red) versions of the risk model. The storm maximum power dissipation is the sum over all storms of the cube of their lifetime maximum wind speeds. The square of the correlation coefficient between the risk model results and the best track data are shown in the upper left of each panel

Table 1 Models used in this study

\begin{tabular}{|c|c|c|c|}
\hline Modeling center & Institute ID & Model name & $\begin{array}{l}\text { Average horizontal resolution } \\
\text { (degrees longitude } \times \text { degrees } \\
\text { latitude) }\end{array}$ \\
\hline $\begin{array}{l}\text { NOAA Geophysical Fluid Dynamics } \\
\text { Laboratory }\end{array}$ & GFDL & CM3 & $2.5 \times 2.0$ \\
\hline UK Met Office Hadley Center & HadGEM & HadGEM2-ES & $1.875 \times 1.25$ \\
\hline Institut Pierre-Simon Laplace & IPSL & CM5A-LR & $3.75 \times 1.89$ \\
\hline $\begin{array}{l}\text { Atmosphere and Ocean Research } \\
\text { Institute (The University of Tokyo), } \\
\text { National Institute for Environmental } \\
\text { Studies, and Japan Agency for Marine- } \\
\text { Earth Science and Technology }\end{array}$ & MIROC & MIROC-5 & $1.4 \times 1.4$ \\
\hline Max Planck Institute for Meteorology & MPI & MPI-ESM-MR & $1.88 \times 1.86$ \\
\hline Meteorological Research Institute & MRI & MRI-CGCM3 & $1.12 \times 1.12$ \\
\hline $\begin{array}{l}\text { National Center for Atmospheric } \\
\text { Research }\end{array}$ & NCAR & CCSM4 & $1.25 \times 0.94$ \\
\hline
\end{tabular}

The evolutions over time of the mean and standard deviations among the 7 models of the global frequency of tropical cyclones and the power dissipation index are shown, respectively, in Figs. 8 and 9. For each model, the seeding rate has been adjusted to yield 80 tropical cyclones per year averaged over the period 1950-2004.

The FAST version of the risk model produces only a minor and perhaps not significant increase in the global frequency of tropical cyclones compared to the original CHIPS version which, as noted in (Emanuel 2013), produces a robust increase of about $25 \%$ in the global number of events. There is also somewhat more scatter among the 7 models in the FAST case, indicating a greater sensitivity to the model used.

The power dissipation index increases by roughly the same percentage in the FAST simulations as in the original CHIPS version, but here again there is more scatter among the models downscaled. 

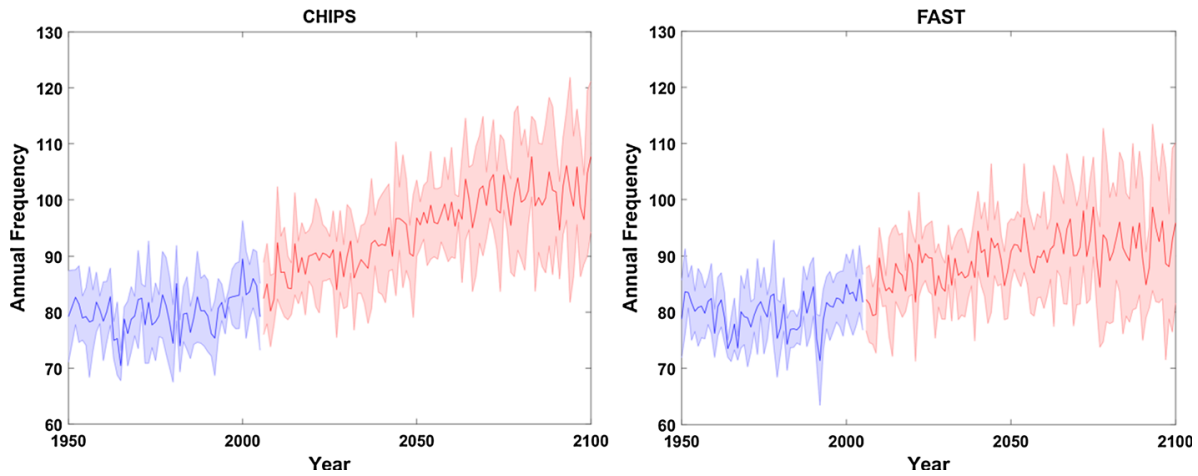

Fig. 8 Evolution of the global frequency of tropical cyclones according to the CHIPS (left) and FAST (right) versions of the risk model. The blue corresponds to the CMIP5 historical period 1950-2005, while the red denotes the years 2006-2100 under Representative Concentration Pathway (RCP) 8.5. The solid curve shows the 7-model mean, while the shading represents one standard deviation up and down from the mean
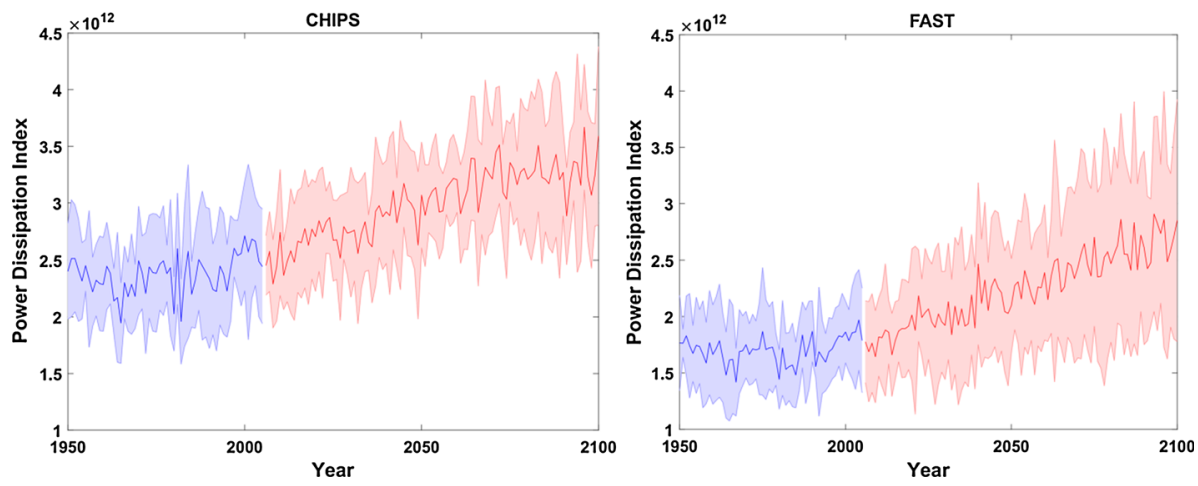

Fig. 9 Same as Fig. 8 but for the power dissipation index (in $\mathrm{m}^{3} \mathrm{~s}^{-2}$ )

Of course, in this case, we do not know what the "right" answer is, but note that the FAST result is closer to the current consensus that global tropical cyclone frequency should remain constant or decrease slightly as the planet warms. This consensus is based mostly on tropical cyclones simulated explicitly but crudely in global climate models, but scaling arguments and limited area cloud-resolving simulations also suggest a decrease in frequency (Khairoutdinov and Emanuel 2013).

\section{Summary}

We developed a fast tropical cyclone intensity simulator that can be used to rapidly estimate storm intensity along a specified tropical cyclone track, given estimates of the potential intensity, 250-850 hPa environmental wind shear, and upper ocean properties along the track. It is much faster than the CHIPS model but does not explicitly simulate any facet of storm structure, which must be parameterized. The model consists of a pair of ordinary differential equations that can be rapidly coded and solved. While motivated by 
theory and existing models, the new simulator should be considered an empirical construct, developed to mimic the behavior of individual storms and of a risk model based on CHIPS, driven by the same environmental parameters as the latter.

In a small number of hindcasts of Atlantic hurricanes, the performance of the intensity simulator is comparable to that of the full CHIPS model (Fig. 2). When used to simulate the climatology of tropical cyclones, there are notable differences between the intensity simulator and CHIPS (Figs. 3, 4, 5, 6, 7) but it is difficult to judge which technique is superior. Future work will examine the differences between the two techniques in other ocean basins.

When forced by an evolving climate simulated by 7 CMIP5 models, the new simulator shows less sensitivity of global tropical cyclone frequency to increasing temperature but about the same increase in global tropical cyclone power dissipation (Figs. 8, 9).

We hope that this simple model will facilitate estimation of long-term tropical cyclone risk and stimulate further work on reduced tropical cyclone intensity models.

Open Access This article is distributed under the terms of the Creative Commons Attribution 4.0 International License (http://creativecommons.org/licenses/by/4.0/), which permits unrestricted use, distribution, and reproduction in any medium, provided you give appropriate credit to the original author(s) and the source, provide a link to the Creative Commons license, and indicate if changes were made.

\section{Appendix}

\section{Inclusion of dissipative heating and the pressure dependence of the saturation specific humidity}

We begin with the original formulation of Emanuel (2012), which did not include effects of dissipative heating or the pressure dependence of the saturation mixing ratio in the inflowing air:

$$
\frac{\mathrm{d} V}{\mathrm{~d} t} \cong \frac{C_{D}}{2 h}\left(V_{p 0}^{2}-V^{2}\right)
$$

where $V_{p 0}$ is the potential intensity defined without including either isothermal expansion or dissipative heating. According to Emanuel and Rotunno (2011) (see their equations 40 and 41), this unmodified potential intensity is given by

$$
V_{p 0}^{2}=\frac{C_{k}}{C_{\mathrm{D}}} F\left(\frac{C_{k}}{C_{\mathrm{D}}}\right) \epsilon\left(h_{0 e}^{*}-h_{e}^{*}\right),
$$

where $C_{k}$ is the surface enthalpy coefficient, $h_{0 e}^{*}$ is the saturation moist static energy of the unperturbed sea surface, $h_{e}^{*}$ is the saturation moist static energy of the unperturbed free troposphere (which, in a moist adiabatic atmosphere, is independent of height), taken to be equal to the actual moist static energy of the subcloud layer in a convectively equilibrated atmosphere, and $F$ is a function of the ratio of the surface exchange coefficients given in Emanuel and Rotunno (2011).

If we multiply (10) by $V$ and substitute (11), the result is

$$
h \frac{\mathrm{d} V^{2}}{\mathrm{~d} t}=C_{k} F \epsilon V\left(h_{0 e}^{*}-h_{e}^{*}\right)-C_{\mathrm{D}} V^{3} .
$$


This is the kinetic energy equation of the system. The first term on the right is the generation of mechanical energy by the hurricane's Carnot cycle driven by surface enthalpy fluxes, and $F$ essentially converts the environmental thermodynamic disequilibrium, $h_{0}^{*}-h_{e}^{*}$, into the local eyewall thermodynamic disequilibrium, $h_{0}^{*}-h_{\text {eyewall }}^{*}$, where $h_{\text {eyewall }}^{*}$ is the tropospheric saturation moist static energy in the eyewall. The last term in (12) is the dissipation of kinetic energy by boundary layer turbulence.

Dissipative heating adds to (12) the term $\epsilon C_{D} V^{3}$ (Bister and Emanuel 1998), reflecting that dissipative heating increases with the cube of the wind speed. Accounting for this and the pressure dependence of the surface saturation moist static energy, (12) can be written

$$
h \frac{\mathrm{d} V^{2}}{\mathrm{~d} t}=C_{k} F \epsilon V\left(h_{0 \text { eyewall }}^{*}-h_{e}^{*}\right)-C_{\mathrm{D}}(1-\epsilon) V^{3},
$$

where $h_{\text {Oeyewall }}^{*}$ is the saturation moist static energy at the radius of maximum wind.

To account for the pressure dependence of the surface saturation moist static energy, we write

$$
h_{\text {Oeyewall }}^{*}-h_{0 e}^{*}=L_{\mathrm{v}} q_{0 e}^{*}\left(\frac{p_{0}}{p_{\text {eyewall }}}-1\right) \cong L_{\mathrm{v}} q_{0 e}^{*} \ln \left(\frac{p_{0}}{p_{\text {eyewall }}}\right),
$$

where $L_{\mathrm{v}}$ is the latent heat of vaporization, $q_{0 e}^{*}$ is the saturation specific humidity of the unperturbed sea surface, $p_{0}$ is the unperturbed surface pressure, and $p_{\text {eyewall }}$ is the surface pressure under the eyewall. The term results from the approximation that the fractional pressure drop to the eyewall is small, applied to the definitions of moist static energy and saturation specific humidity

We next relate the pressure drop under the eyewall to the maximum circular wind speed, $V$. There are quite a few published empirical relationships between central pressure and maximum wind, but here we seek a relationship for the eyewall rather than the central pressure.

We first point out that were the boundary layer flow steady and frictionless, the Bernoulli equation, integrated from the outer radius of vanishing wind to the radius of maximum winds, would have the form

$$
\int_{e}^{\text {eyewall }} \frac{d p}{\rho}=\int_{e}^{\text {eyewall }} R_{d} T_{s} d \ln (p)=R_{d} T_{s} \ln \left(\frac{p_{\text {eyewall }}}{p_{0}}\right)=-\frac{1}{2} V^{2},
$$

where we have used the ideal gas law in substitution for the density $\rho$, and $R_{d}$ is the gas constant for dry air. This should be regarded as a lower bound on the magnitude of the pressure drop, as frictional dissipation will increase the pressure drop relative to the maximum wind speed. This suggests that the pressure drop scales as $V^{2} / R_{d} T_{s}$. A curve fit to the output of the full CHIPS model, in which the radial pressure distribution is calculated from gradient wind balance, yields

$$
\ln \left(\frac{p_{0}}{p_{\text {eyewall }}}\right) \cong \frac{1}{2} \frac{V^{2}}{R_{d} T_{s}}
$$

While this is not a highly accurate predictor of eyewall pressure, it should suffice for the present purpose, given the simplicity of the model.

Substituting (16) into (14) and the result into (13) yields 


$$
h \frac{\mathrm{d} V^{2}}{\mathrm{~d} t}=C_{k} F \epsilon V\left(h_{0 e}^{*}-h_{e}^{*}\right)-C_{\mathrm{D}}(1-\varepsilon-\kappa) V^{3}=C_{\mathrm{D}} V\left[V_{p 0}^{2}-(1-\varepsilon-\kappa) V^{2}\right],
$$

where $V_{p 0}^{2}$ is given by (11) and

$$
\kappa \equiv \frac{C_{k}}{C_{\mathrm{D}}} F\left(\frac{C_{k}}{C_{\mathrm{D}}}\right) \epsilon \frac{L_{\mathrm{v}} q_{0}^{*}}{2 R_{d} T_{s}} .
$$

In the algorithm used to calculate potential intensity, we take $F=1$, lacking good quantitative estimates of the exchange coefficients at high wind speed.

Before taking the final step in this derivation, we pause to consider the effects of ocean interaction. We will do that through the feedback factor $\alpha$ defined by (4) in the main text. But note that this is a factor that multiplies the surface enthalpy flux, which we can incorporate by multiplying the enthalpy exchange coefficient $C_{k}$ by $\alpha$ in the derivation (12)-(17). The result is that (17) is rewritten

$$
\frac{\mathrm{d} V}{\mathrm{~d} t}=\frac{C_{\mathrm{D}}}{2 h}\left[\alpha V_{p 0}^{2}-(1-\epsilon-\alpha \kappa) V^{2}\right] .
$$

Clearly the actual potential intensity achieved when $\alpha=1$ in (19) is given by

$$
V_{p}^{2}=\frac{V_{p 0}^{2}}{1-\epsilon-\kappa} .
$$

Since existing algorithms generate the full potential intensity, we prefer to use that rather than the form that does not include dissipative heating or the pressure dependence of the surface saturation specific humidity. Thus, we use (20) to rewrite (19) as

$$
\frac{\mathrm{d} V}{\mathrm{~d} t}=\frac{C_{\mathrm{D}}}{2 h}\left[\alpha(1-\epsilon-\kappa) V_{p}^{2}-(1-\epsilon-\alpha \kappa) V^{2}\right] .
$$

Finally, the enthalpy increase in the boundary layer cannot be communicated to the free troposphere if the latter is dry. This includes the enthalpy increase from dissipative heating. Thus, we multiply all the terms, except for the spin-down term $-V^{2}$, in (21), by the cube of the inner core moisture variable, $m$. This results in Eq. (2) in the main text. The choice of the exponent 3 by which $m$ is raised is empirical, giving a reasonably good fit to the behavior of the full CHIPS model.

\section{References}

Bister M, Emanuel KA (1998) Dissipative heating and hurricane intensity. Meteorol Atmos Phys 50:233-240

Colbert AJ, Soden BJ, Vecchi GA, Kirtman BP (2013) The impact of anthropogenic climate change on North Atlantic tropical cyclone tracks. J Clim 26:4088-4095

Emanuel K (2012) Self-stratification of tropical cyclone outflow: part II: implications for storm intensification. J Atmos Sci 69:988-996

Emanuel K (2013) Increased global tropical cyclone activity from global warming: results of downscaling CMIP5 climate models. Proc Nat Acad Sci 110:12219-12224. doi:10.1073/pnas.1301293110

Emanuel K, Jagger TH (2010) On estimating hurricane return periods. J Appl Meteorol Clim 49:837-844

Emanuel K, Rotunno R (2011) Self-stratification of tropical cyclone outflow. Part I: implications for storm structure. J Atmos Sci 68:2236-2249

Emanuel K, Zhang F (2016) On the predictability and error sources of tropical cyclone intensity forecasts. J Atmos Sci 73:3739-3747. doi:10.1175/JAS-D-16-0100.1 
Emanuel K, Zhang F (2017) The role of inner core moisture in tropical cyclone predictability and practical forecast skill. J Atmos Sci. doi:10.1175/JAS-D-17-0008.1

Emanuel K, DesAutels C, Holloway C, Korty R (2004) Environmental control of tropical cyclone intensity. J Atmos Sci 61:843-858

Emanuel KA, Ravela S, Vivant E, Risi C (2006) A statistical-deterministic approach to hurricane risk assessment. Bull Am Meteorol Soc 19:299-314

Emanuel K, Sundararajan R, Williams J (2008) Hurricanes and global warming: results from downscaling IPCC AR4 simulations. Bull Am Meteorol Soc 89:347-367

EM-DAT (2016) The OFDA/CRED International Disaster Database. http://www.emdat.be/

Kalnay E et al (1996) The NCEP/NCAR 40-year reanalysis project. Bull Am Meteorol Soc 77:437-471

Khairoutdinov MF, Emanuel K (2013) Rotating radiative-convective equilibrium simulated by a cloudresolving model. J Adv Model Earth Syst 5:816-825. doi:10.1002/2013MS000253

Levitus S (1982) Climatological atlas of the world ocean. U.S. Government. Print. Off, Washington

Marks DG (1992) The beta and advection model for hurricane track forecasting. Natl. Meteor. Center, Camp Springs, Maryland

Schade LR, Emanuel KA (1999) The ocean's effect on the intensity of tropical cyclones: results from a simple coupled atmosphere-ocean model. J Atmos Sci 56:642-651

Tang B, Emanuel K (2010) Midlevel ventilation's constraint on tropical cyclone intensity. J Atmos Sci 67:1817-1830

Vickery PJ, Skerjl PF, Twisdale LA (2000) Simulation of hurricane risk in the U. S. using empirical track model. J Struct Eng 126:1222-1237

Yonekura E, Hall TM (2011) A statistical model of tropical cyclone tracks in the Western North Pacific with ENSO-dependent cyclogenesis. J Appl Meteorol Clim 50:1725-1739 